\title{
RR Lyrae stars in the Bootes structure
}

\author{
M. Dall'Ora ${ }^{1}$, G. Clementini ${ }^{2}$, K. Kinemuchi ${ }^{3}$, V. Ripepi ${ }^{1}$, \\ M. Marconi ${ }^{1}$, M. Di Criscienzo ${ }^{1}$, L. Di Fabrizio ${ }^{4}$, C. Greco ${ }^{2}$, \\ C. T. Rodgers ${ }^{3}$, C. Kuehn ${ }^{5}$ and H. A. Smith ${ }^{5}$ \\ ${ }^{1}$ INAF - Osservatorio Astronomico di Capodimonte, via Moiariello 16, I-80131, Napoli, Italy \\ email: dallora,ripepi,marconi,dicrisci@oacn.inaf.it \\ ${ }^{2}$ INAF - Osservatorio Astronomico di Bologna, via Ranzani 1, I-40127, Bologna, Italy \\ email: gisella.clementini,claudia.greco@oabo.inaf.it \\ ${ }^{3}$ University of Wyoming, Department of Physics and Astronomy, Department 3905, Laramie, \\ WY 82071 \\ email:kinemuch,crodgers@uwyo.edu \\ ${ }^{4}$ INAF, Centro Galileo Galilei and Telescopio Nazionale Galileo, P.O. Box 565, 38700 Santa \\ Cruz de La Palma, Spain \\ email: difabrizio@tng.iac.es \\ ${ }^{5}$ Department of Physics and Astronomy, Michigan State University, East Lansing, MI \\ 48824-2320, email:kuehn@pa.msu.edu,smith@pa.msu.edu
}

\begin{abstract}
V, I$ time-series photometry of the new Milky Way satellite recently discovered in the Bootes constellation (Belokurov et al. 2006) was collected at the TNG, WIRO and Loiano telescopes and used to search for variable stars. We have identified a non-negligible population of RR Lyrae variables, which confirms the presence of an old population in this newly discovered structure. The distribution of periods coupled with information on the evolutionary status of the system derived fromthe color-magnitude diagram provide important hints on the nature and history of this intriguing new companion of the Milky Way.
\end{abstract}

Keywords. galaxies: distances and redshifts - galaxies: dwarf - galaxies: individual (Bootes) stars: horizontal-branch - stars: variables: other - techniques: photometric

\section{Bootes: a new faint companion of the Milky Way}

In the framework of an extensive search for new objects in the Galactic halo, a new satellite of the Milky Way was recently discovered by Belokurov et al. (2006) in the constellation of Bootes. Photometric observations suggest that Bootes is an old, very metal-poor dwarf spheroidal galaxy (dSph), and spectroscopy of Red Giants belonging to the AGB (Munoz et al. 2006) confirmed a very low metal content of the order of $[\mathrm{Fe} / \mathrm{H}] \sim$ -2.5 , making Bootes the most metal-poor Local Group dSph known so far. The presence of a well extended Horizontal Branch (HB) clearly crossing the Instability Strip (in the $i,(g-i)$ color-magnitude diagram $(\mathrm{CMD}))$, suggests that a non-negligible population of RR Lyrae stars should be present in Bootes. RR Lyre stars are well-established standard candles and tracers of old stellar populations. Their pulsation properties can thus be used to derive useful hints and constraints on the evolutionary history of the parent population.

\section{The RR Lyrae population of the Bootes dSph}

Siegel (2006, hereafter S06) reported the discovery of 15 RR Lyrae stars in the Bootes dSph. Independently, our group has collected time-series photometry in the $V, I$ bands, 

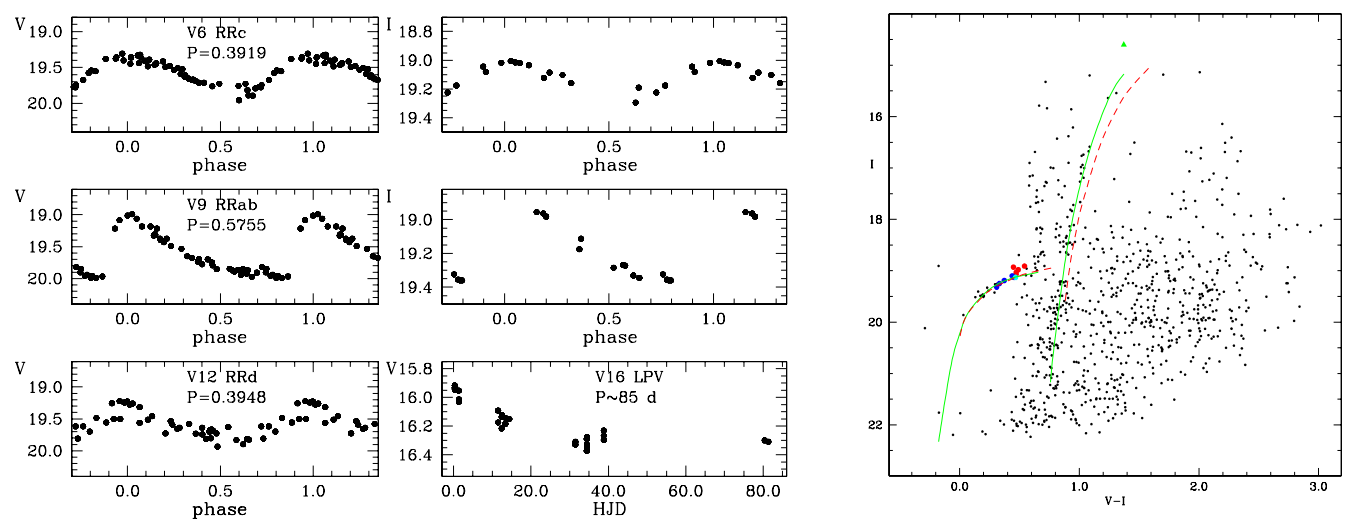

Figure 1. Left panel: $V, I$ light curves for the RRc star V6 and the RRab star V9. $V$-band light curves of the RRd pulsator V12, and of the LPV variable star V16. Right panel: $I, V-I$ color-magnitude diagram of the Bootes dSph. Filled circles are RR Lyrae stars, the filled triangle marks the long period variable. Solid and dashed lines show the mean ridge lines of M15 and M3, respectively, shifted in magnitude and adjusted in reddening to fit the galaxy HB.

with the aim of discovering and fully characterizing the Bootes variable stars population. Observations were obtained with the BFOSC camera available at the $1.52 \mathrm{~m}$ of the INAFOAB Telescope in Loiano, with DOLORES at the 3.5m INAF-TNG telescope in La Palma, and with the WIRO-Prime camera at the Wyoming Infared Observatory $2.3 \mathrm{~m}$ telescope. We identified 12 variable stars belonging to Bootes $\mathrm{dSph}, 11$ of which are bona fide RR Lyrae stars (all of them are in common with S06), and one Long Period Variable (LPV, see Figure 1, right panel). Their positions on the galaxy CMD (see Figure 1, left panel) strongly suggest their membership to the Bootes dSph. Five variables are fundamental mode pulsators (RRab), five are first-overtones (RRc), and one is a mixedmode pulsator (RRd). The mean period of the RRab stars: $<$ Pab $>=0.69 \mathrm{~d}$, and the high percentage of RRc stars are consistent with Bootes being a pure Oosterhoff type II system. Along with the low metal abundance, these features make the Bootes dSph an analog of the Ursa Minor dSph.

The distance to the Bootes $\mathrm{dSph}$ has been estimated using three different methods based on the RR Lyrae star properties, leading to a weighted mean modulus of $\mu_{0}=$ $19.14 \pm 0.07$ mag. Details can be found in Dall'Ora et al. (2006).

\section{References}

Belokurov, V., et al. 2006, ApJ 647, L111

Dall'Ora, M., et al. 2006, ApJ 653, L109

Munoz, R.R., et al. 2006, ApJ 650, L51

Siegel, M.H. 2006, $A p J$ 649, L83 